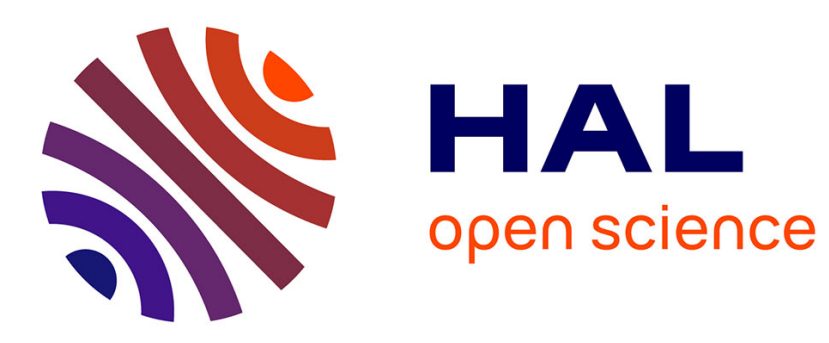

\title{
Arc à cathode creuse : étude expérimentale de la colonne de plasma à l'intérieur de la cathode
}

\author{
A. Brunet
}

\section{To cite this version:}

A. Brunet. Arc à cathode creuse: étude expérimentale de la colonne de plasma à l'intérieur de la cathode. Revue de Physique Appliquée, 1977, 12 (8), pp.1105-1110. 10.1051/rphysap:019770012080110500 . jpa-00244286

\section{HAL Id: jpa-00244286 https://hal.science/jpa-00244286}

Submitted on 1 Jan 1977

HAL is a multi-disciplinary open access archive for the deposit and dissemination of scientific research documents, whether they are published or not. The documents may come from teaching and research institutions in France or abroad, or from public or private research centers.
L'archive ouverte pluridisciplinaire HAL, est destinée au dépôt et à la diffusion de documents scientifiques de niveau recherche, publiés ou non, émanant des établissements d'enseignement et de recherche français ou étrangers, des laboratoires publics ou privés. 


\author{
Classification \\ Physics Abstracts \\ 6.700
}

\title{
ARC A CATHODE CREUSE : ÉTUDE EXPÉRIMENTALE DE LA COLONNE DE PLASMA A L'INTÉRIEUR DE LA CATHOde (*)
}

\author{
A. BRUNET \\ Office National d'Etudes et de Recherches Aérospatiales (O. N. E. R. A.), \\ 29, avenue de la division Leclerc, 92320 Châtillon, France
}

(Reçu le 22 décembre 1976, accepté le 29 avril 1977)

\begin{abstract}
Résumé. - On considère un arc à cathode creuse fonctionnant avec un écoulement d'argon. On détermine la répartition de pression à l'intérieur de la cathode. La densité du plasma, la température électronique, le potentiel flottant et le potentiel plasma sont déduits de mesures effectuées au moyen de sondes électrostatiques. A partir de ces données on propose un modèle simplifié de la colonne de plasma qui se trouve à l'intérieur de la cathode. Le courant et le bilan d'énergie calculés à la surface de la cathode avec ce modèle correspondent bien aux valeurs mesurées.
\end{abstract}

\begin{abstract}
We consider a hollow cathode arc working with an argon flow. The pressure distribution is determined inside the cathode. The plasma density, electron temperature, floating potential and plasma potential are deduced from electrostatic probe measurements. From these data a simplified model of the plasma column into the cathode is given. The current and the energy balance calculated at the surface of the cathode with this model are in good agreement with the measurements.
\end{abstract}

1. Introduction. - Nous considérons une décharge à cathode creuse fonctionnant en régime d'arc dans l'argon. Celui-ci est introduit en permanence à l'intérieur de la cathode et absorbé par un groupe de pompage. Depuis les premiers travaux de Luce et Lidsky $[1,2]$ de nombreux auteurs ont étudié ces décharges, mais ils se sont surtout intéressés à la colonne de plasma qui se trouve entre la cathode et l'anode [3]. La colonne de plasma ne se limite pas à l'espace cathode anode mais pénètre à l'intérieur de la cathode. Nous avons déterminé la densité des neutres, des ions, des électrons et la température électronique à l'intérieur de celle-ci. La connaissance de ces paramètres a permis de préciser les mécanismes de production du plasma et de définir un modèle physique de la décharge à l'intérieur de la cathode. Parmi les applications de ces décharges nous citerons : les sources d'ions, la propulsion ionique où elles peuvent être utilisées comme source ou comme neutraliseur, certains lasers. En France, elles ont été particulièrement étudiées au Laboratoire de Physique des Plasmas d'Orsay.

2. Dispositif expérimental. - La cathode qui est portée à haute température doit être réalisée en métal

(*) Communication présentée au Congrès National de Physique des Plasmas, Paris, 6-10 décembre 1976. réfractaire. Le tantale a été préféré au tungstène parce qu'il est beaucoup plus facile à travailler que ce dernier. Pour pouvoir faire des mesures à l'intérieur de la cathode à l'aide de sondes, il était nécessaire d'utiliser des cathodes de diamètre suffisamment grand. D'un autre côté il fallait que les débits de gaz restent compatibles avec la capacité des groupes de pompage. Pour satisfaire ces deux conditions, on a choisi des cathodes de $6 \mathrm{~mm}$ de diamètre extérieur et $0,2 \mathrm{~mm}$ d'épaisseur. L'anode est une plaque de tantale percée d'un trou de $10 \mathrm{~mm}$ de diamètre ; elle est placée à $8 \mathrm{~mm}$ en aval du plan de sortie de la cathode. L'enceinte à vide est un cylindre de pyrex de $30 \mathrm{~cm}$ de diamètre et $60 \mathrm{~cm}$ de long, fermé par 2 disques en dural (Fig. 1). Lorsque la décharge fonctionne la cathode est portée à une température de l'ordre de $2000^{\circ} \mathrm{C}$ sur une longueur de quelques centimètres à partir de son extrémité. Il existe un maximum de température en amont de l'extrémité de la cathode ; la position de ce maximum et la longueur de la zone incandescente, que nous désignerons par la suite sous le nom de zone active, varient avec le débit d'argon. Les débits sont compris entre $10^{-4}$ et $5 \times 10^{-2} \mathrm{~g} / \mathrm{s}$ et pour ceux-ci la pression dans l'enceinte est inférieure à 10 Pascal. Les courants de décharge varient entre 5 et $30 \mathrm{~A}$ et les courbes $I=f(V)$ présentent une pente négative caractéristique du régime d'arc. 


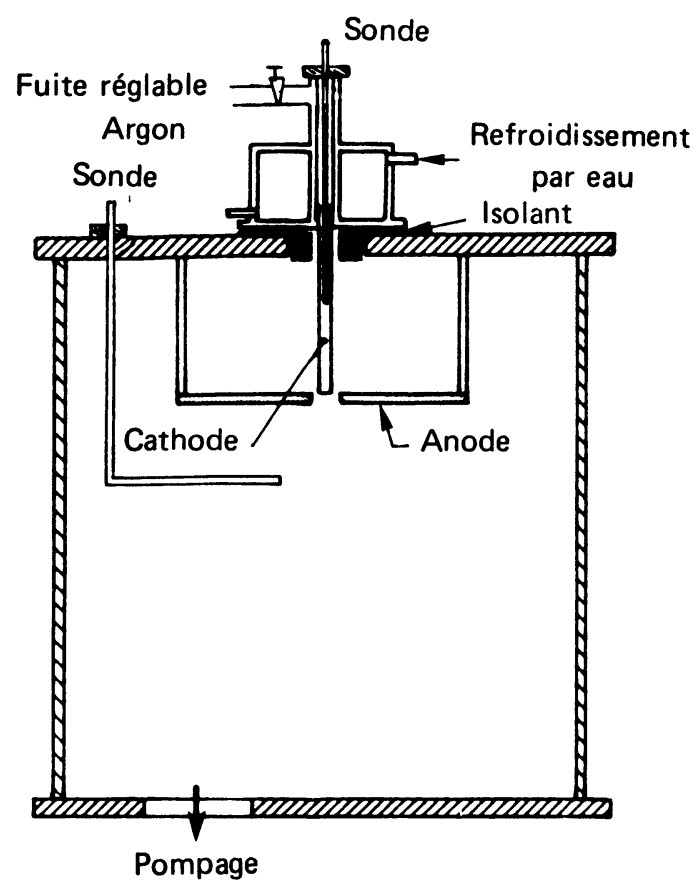

FIG. 1. - Dispositif expérimental. [Experimental device.]

3. Pression à l'intérieur de la cathode. - Le calcul des conditions de l'écoulement à l'intérieur de la cathode ainsi que les mesures de pression faites dans celle-ci montrent qu'il existe un col sonique à l'extrémité de la cathode $[4,5]$. Dans ces conditions le débit de gaz est indépendant de la pression dans l'enceinte. La pression dans le plan de sortie de la cathode ne dépend que du débit et de la température du gaz. Le calcul du transfert de chaleur entre le gaz et la paroi de la cathode montre qu'avec les débits d'argon utilisés ici la température du gaz est très voisine de celle de la paroi. C'est à l'entrée de la zone active que l'écart de température entre le gaz et la paroi est maximal et même pour les débits les plus élevés il reste inférieur à $100^{\circ} \mathrm{C}$. En première approximation on peut donc admettre que la température du gaz est égale à celle de la paroi. La température de la paroi est mesurée avec un pyromètre optique dans la zone active et avec des thermocouples en amont de celle-ci. Dans le plan de sortie de la cathode la vitesse du son $v_{\mathrm{s}}$ est égale à :

$$
V_{\mathrm{s}}=\sqrt{\gamma \frac{k T}{m}}
$$

avec $k=$ constante de Boltzmann,

$T=$ température du gaz,

$m=$ masse de l'atome d'argon,

$\gamma=$ rapport des chaleurs spécifiques.

Pour un débit $\stackrel{\circ}{m}$ et une vitesse uniforme dans toute la section $\sigma$ de la cathode la pression $p_{\mathrm{s}}$ dans le plan de sortie est égale à :

$$
p_{\mathrm{s}}=\frac{\stackrel{\circ}{m}}{\sigma} \sqrt{\frac{k T}{\gamma_{m}}} .
$$

En réalité, l'écoulement est laminaire et la vitesse dans une section droite n'est pas uniforme mais varie selon une loi parabolique, si on néglige l'apport de chaleur à la paroi (température du gaz = température de la paroi). Compte tenu de ceci l'expression précédente doit s'écrire :

$$
p_{\mathrm{s}}=\frac{2 \stackrel{\circ}{m}}{\sigma} \sqrt{\frac{k T}{\gamma_{m}}} .
$$

On calcule donc, pour un débit donné, la pression dans le plan de sortie de la cathode. A partir de là, connaissant la température en fonction de l'abscisse, on calcule la pression à l'intérieur de la cathode. Les valeurs calculées de cette façon ont été comparées aux valeurs mesurées $10 \mathrm{~cm}$ en amont du plan de sortie de la cathode et les résultats sont en bon accord. Sur la figure 2 sont présentées les courbes qui donnent

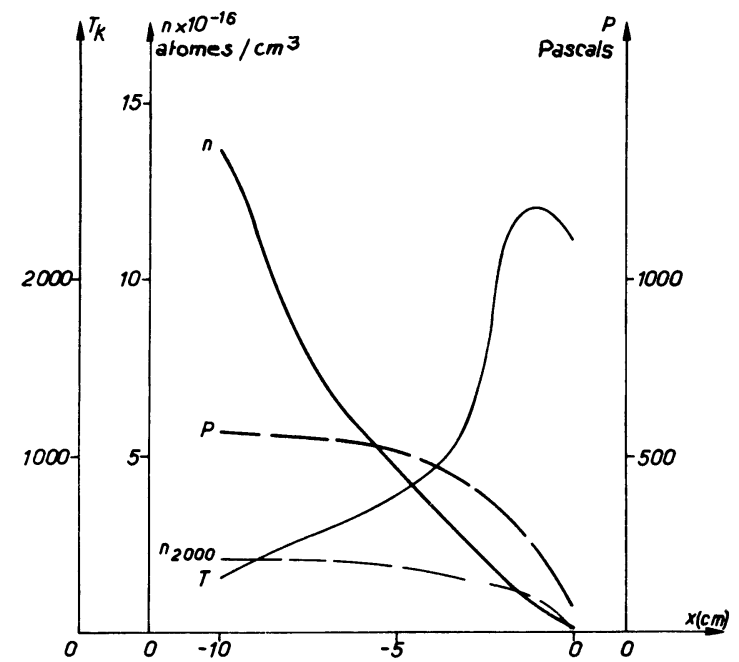

FIg. 2. - Répartition de la température, de la pression, de la densité des neutres en fonction de l'abscisse. La courbe $\mathbf{n}_{2000}$ représente la variation de la densité des neutres dans le cas où la température de l'argon serait constante et égale à $2000^{\circ}$.

[Temperature, pressure, density of atom distribution as a function of abscissa. The curve $\mathrm{n}_{2000}$ gives the change in atom density for a constant argon temperature of $2000^{\circ}$.]

la pression, la densité de neutres calculée par cette méthode, et la température mesurée sur la paroi. La courbe $\mathrm{n}_{2000}$ représente, à titre de comparaison, la variation de la densité d'atomes d'argon dans le cas d'un écoulement isotherme à $2000^{\circ} \mathrm{C}$. On remarque que, dans le cas que nous considérons, du fait de l'apport de chaleur, la densité d'atomes neutres dans la zone active décroit beaucoup plus rapidement.

4. Mesure du potentiel et de la densité du plasma à l'intérieur de la cathode. - Pour déterminer les caractéristiques du plasma à l'intérieur de la cathode nous avons utilisé des sondes électrostatiques. La cathode ne mesurant que $5,6 \mathrm{~mm}$ de diamètre intérieur, les sondes doivent être suffisamment petites pour ne pas perturber les conditions d'écoulement. L'isolant 
qui enrobe la sonde doit pouvoir supporter les températures très élevées qui existent à l'intérieur de la cathode et pour cela nous avons dû utiliser du nitrure de bore. La sonde est un fil de tungstène de $0,2 \mathrm{~mm}$ de diamètre et son support est constitué d'une tige de nitrure de bore de $1,4 \mathrm{~mm}$ de diamètre. La sonde est introduite par l'arrière de la cathode et il est possible de la déplacer sur l'axe de celle-ci. Une deuxième sonde placée dans l'enceinte permet de faire des mesures sur le plasma éjecté hors de la cathode (Fig. 1).

Lorsque la sonde est enfoncée à l'intérieur de la cathode, elle collecte un courant dès qu'elle se trouve à $2 \mathrm{~cm}$ environ du début de la zone active. Ce courant est faible (de l'ordre des microampères) et il est dû à des électrons qui diffusent dans le gaz, dans la direction opposée à l'écoulement. Ensuite, on relève des caractéristiques de sonde à partir de la limite amont de la zone active mais il n'est pas possible d'explorer celle-ci sur toute sa longueur. Il existe une limite aval pour le déplacement de la sonde. Lorsqu'on dépasse celle-ci les caractéristiques de sonde sont perturbées et des instabilités apparaissent. Celles-ci sont dues aux électrons émis par la paroi de la cathode qui arrivent jusque sur l'axe lorsque la pression est suffisamment basse.

Avec les sondes on détermine le potentiel flottant $V_{\mathrm{f}}$, la température électronique $T_{\mathrm{e}}$ et la densité du plasma $n$ (Fig. 3, 4). La température électronique est

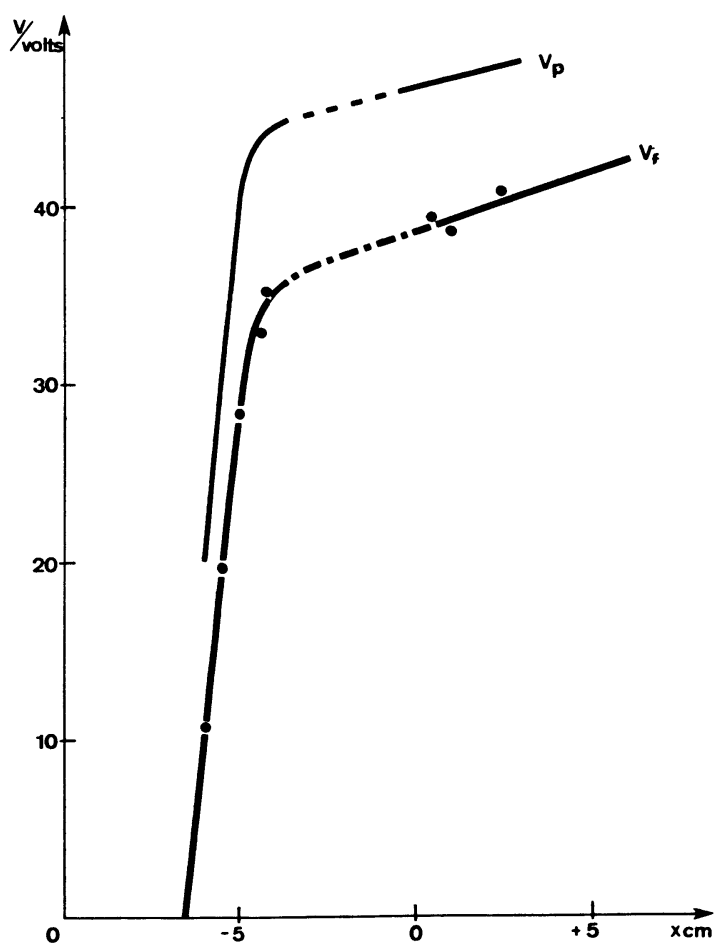

FIG. 3. - Potentiels flottants $V_{\mathrm{f}}$ mesurés sur l'axe de la cathode. Potentiels plasmas $V_{\mathrm{p}}$ calculés à partir des potentiels flottants et des températures électroniques $\left(\stackrel{\circ}{m}=1,2 \times 10^{\mathrm{m}_{3}} \mathrm{~g} / \mathrm{s}, I=10 \mathrm{~A}\right.$, $V=50 \mathrm{~V}$ ).

[Floating potentials $V_{\mathrm{f}}$ measured on the cathode axis. Plasma potentials $V_{\mathrm{p}}$ calculated from the floating potentials and the electron temperatures $\left(\stackrel{\circ}{m}=1.2 \times 10^{-3} \mathrm{~g} / \mathrm{s} ; I=10 \mathrm{~A} ; V=50 \mathrm{~V}\right)$.]

REVUE DB PHYSIQUe APPLIQUée. - T. 12, N• 8, AOUT 1977 de $2 \mathrm{eV}$. Le potentiel plasma $V_{\mathrm{p}}$ est déterminé à partir du potentiel flottant et de la température des électrons. La densité électronique augmente très rapidement à l'arrière de la zone active. Elle croît de 3 ordres de grandeur en $5 \mathrm{~mm}$. La densité du plasma dans la zone active est de l'ordre de $10^{14}$ électrons $/ \mathrm{cm}^{3}$. Sur l'axe, le champ électrique est faible $(\simeq 1 \mathrm{~V} / \mathrm{cm})$ et le potentiel plasma n'est inférieur que de quelques volts au poteniiel de l'anode.

Dans le plan de sortie de la cathode on suppose que le courant est parallèle à l'axe et qu'il n'est transporté que par les électrons, la mobilité de ceux-ci étant beaucoup plus grande que celle des ions, on peut alors écrire la densité de courant :

$$
\begin{aligned}
q & =\text { charge de l'électron, } \\
j=n q v_{\mathrm{d}} \quad v_{\mathrm{d}} & =\text { vitesse dirigée des électrons, }
\end{aligned}
$$

$j, q, v_{\mathrm{d}}$ sont connus; on peut donc calculer $n$ dans le plan de sortie de la cathode. La valeur obtenue par cette voie est en bon accord avec les valeurs mesurées à l'intérieur de la cathode ou dans l'enceinte à $5 \mathrm{~mm}$ en aval du plan de sortie de la cathode (Fig. 4).

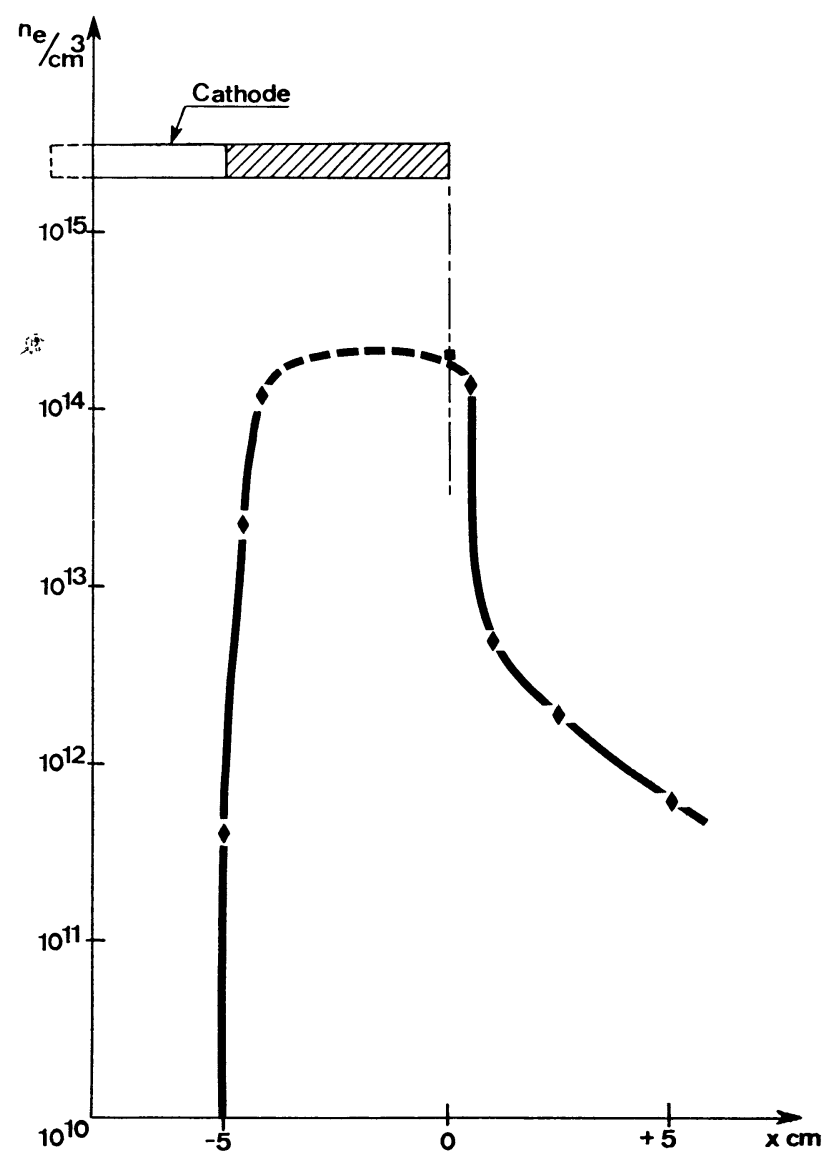

Fig. 4. - Variation de la densité électronique en fonction de l'abscisse $\left(\stackrel{\circ}{m}=1,2 \times 10^{-3} \mathrm{~g} / \mathrm{s} ; I=10 \mathrm{~A} ; V=50 \mathrm{~V}\right)$.

[Variation in electron density as a function of abscissa

$$
\left.\left.\stackrel{\circ}{m}=1.2 \times 10^{-3} \mathrm{~g} / \mathrm{s} ; I=10 \mathrm{~A} ; V=50 \mathrm{~V}\right) .\right]
$$

Les figures 5 et 6 présentent les résultats des mesures de la densité et du potentiel pour différents débits et 


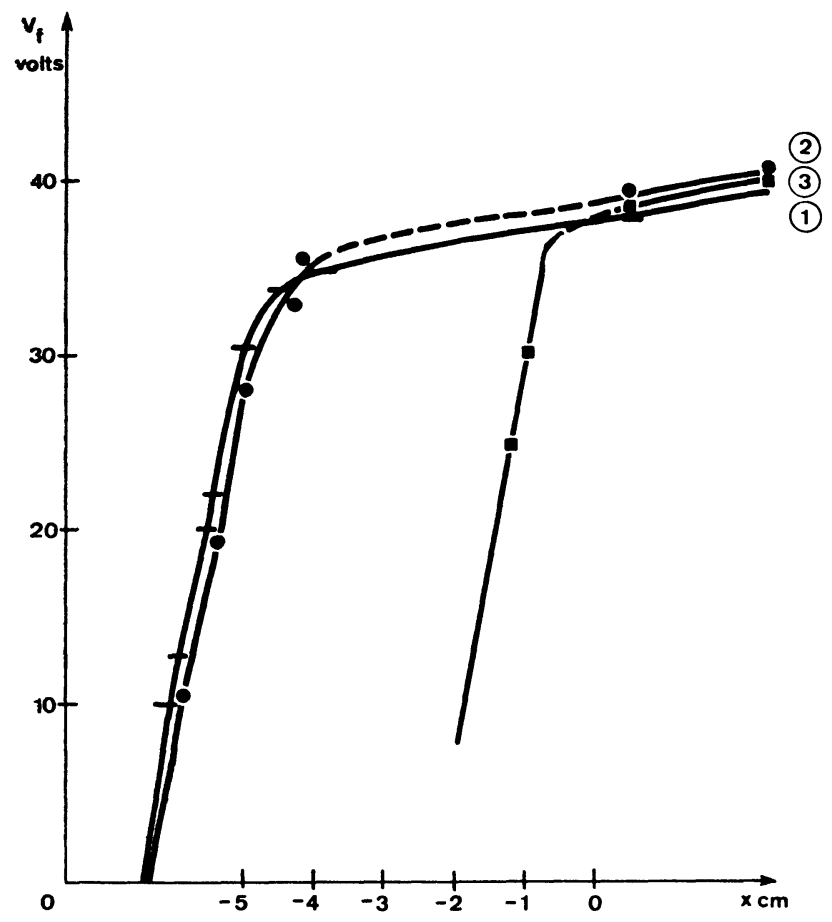

Fig. 5. - Variation de la répartition du potentiel flottant avec le courant et le débit d'argon. $1 . I=15 \mathrm{~A} ; m=1,2 \times 10^{-3} \mathrm{~g} / \mathrm{s}$;

$$
\begin{array}{ll}
\text { 2. } I=10 \mathrm{~A} ; \stackrel{\circ}{m}=1,2 \times 10^{-3} \mathrm{~g} / \mathrm{s} ; \\
\text { 3. } I=10 \mathrm{~A} ; \stackrel{\circ}{m}=3,5 \times 10^{-3} \mathrm{~g} / \mathrm{s} \text {. }
\end{array}
$$

[Variation of the floating potential repartition with the current and the argon mass flow rate. $1 . I=15 \mathrm{~A} ; \stackrel{\circ}{m}=1.2 \times 10^{-3} \mathrm{~g} / \mathrm{s}$

$$
\begin{array}{ll}
\text { 2. } I=10 \mathrm{~A} ; & \stackrel{\circ}{m}=1.2 \times 10^{-3} \mathrm{~g} / \mathrm{s} ; \\
\text { 3. } I=10 \mathrm{~A} ; & \left.\stackrel{\circ}{m}=3.5 \times 10^{-3} \mathrm{~g} / \mathrm{s} .\right]
\end{array}
$$

différents courants. Les courbes ont toutes la même allure et on voit que pour un débit d'argon donné, lorsque le courant varie, la longueur de la colonne de plasma reste constante tandis que la densité du plasma augmente avec le courant. Pour un courant donné, si le débit d'argon croît la longueur de la colonne de plasma diminue alors que la densité reste constante.

5. Modèle simplifié de l'intérieur de la cathode. En se basant sur les mesures de potentiel et de densité, on peut définir un modèle de la colonne de plasma qui se trouve à l'intérieur de la cathode. Les courbes des figures 3 et 4 montrent que sur l'axe le potentiel et la densité sont sensiblement constants. Dans le cas envisagé le potentiel sera pris égal à $+45 \mathrm{~V}$ par rapport à la cathode et la densité égale à $10^{14}$ électrons/ $\mathrm{cm}^{3}$ dans tout le plasma à l'intérieur de la cathode. On néglige ainsi le champ axial qui est nécessaire pour assurer le mouvement des électrons hors de la cathode mais ce champ est faible $(1 \mathrm{~V} / \mathrm{cm})$. Comme nous ne considérerons que les phénomènes d'ionisation à l'intérieur de la cathode, sans nous intéresser au transport du courant entre la cathode et l'anode, nous pouvons supposer constant le potentiel sur l'axe.

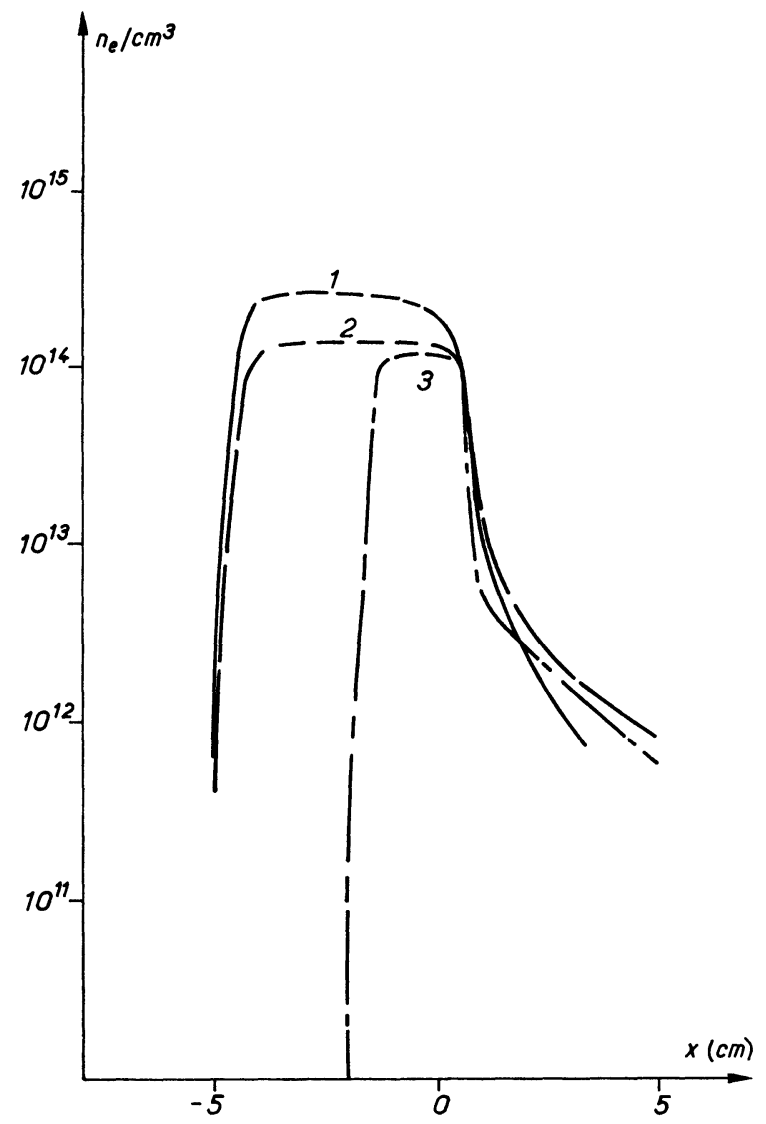

Fig. 6. - Variation de la répartition de densité électronique avec le courant et le débit d'argon. $1 . I=15 \mathrm{~A} ; \stackrel{\circ}{m}=1,2 \times 10^{-3} \mathrm{~g} / \mathrm{s}$;

$$
\begin{aligned}
& \text { 2. } I=10 \mathrm{~A} ; \stackrel{\circ}{m}=1,2 \times 10^{-3} \mathrm{~g} / \mathrm{s} \text {; } \\
& \text { 3. } I=10 \mathrm{~A} ; m=3,5 \times 10^{-3} \mathrm{~g} / \mathrm{s} \text {. }
\end{aligned}
$$

[Variation of the electron density with the current and the argon

$$
\begin{array}{r}
\text { mass flow rate. } 1 . I=15 \mathrm{~A} ; \stackrel{\circ}{m}=1.2 \times 10^{-3} \mathrm{~g} / \mathrm{s} ; \\
\text { 2. } I=10 \mathrm{~A} ; \stackrel{\circ}{m}=1.2 \times 10^{-3} \mathrm{~g} / \mathrm{s} ; \\
\text { 3. } \left.I=10 \mathrm{~A} ; \stackrel{\circ}{m}=3.5 \times 10^{-3} \mathrm{~g} / \mathrm{s} .\right]
\end{array}
$$

5.1 VARIATION RADIALE DU POTENTIEL ET DE LA DENSITÉ. - Etant données les dimensions de la cathode il n'est pas possible de faire une exploration radiale du plasma et il faut déterminer par le calcul la variation radiale du potentiel et de la densité. Pour la diffusion le paramètre caractéristique est le rapport entre la longueur de Debye $L_{\mathrm{D}}$ et le rayon de la cathode [6]. Pour $T_{\mathrm{e}}=15000 \mathrm{~K}$ et $n=10^{14}$ électrons $/ \mathrm{cm}^{3}$, $L_{\mathrm{D}}=8 \times 10^{-5} \mathrm{~cm}$. La longueur de Debye est très inférieure au rayon de la cathode ce qui signifie que la diffusion est ambipolaire. Le calcul de la répartition radiale de densité montre qu'à la limite du plasma la densité est 0,8 fois la densité sur l'axe. Le champ radial est maximal à la limite du plasma et en ce point son amplitude est inférieure à $1 \mathrm{~V} / \mathrm{cm}$ [7]. Sur les $3 \mathrm{~mm}$ que représente le rayon de la cathode la variation de potentiel est donc faible et, en première approximarion, elle sera négligée.

Dans ces conditions, on considérera qu'à l'intérieur 
de la cathode la colonne de plasma est un cylindre de densité et de potentiel constants. Nous avons vu que la différence de potentiel entre le plasma et la cathode était de $+45 \mathrm{~V}$; celle-ci se produit donc dans la gaine qui sépare le plasma de la paroi de la cathode.

5.2 Gaine entre la cathode et le Plasma. A la frontière du plasma et de la gaine on peut décomposer le courant en trois composantes : les ions qui sortent du plasma sous l'effet de l'agitation thermique et qui sont accélérés vers la cathode dans la gaine ; les électrons qui sortent du plasma sous le même effet mais qui sont renvoyés dans celui-ci après avoir été réfléchis dans la gaine où, pour eux, le champ est répulsif ; enfin les électrons émis par la paroi de la cathode qui ont été accélérés dans la gaine. L'épaisseur de la gaine $d$ est calculée en supposant que le courant d'ions y est limité par la charge d'espace. On trouve $d \simeq 10^{-3} \mathrm{~cm}$. Les libres parcours de collision élastique des électrons sont de $10^{-1} \mathrm{~cm}$. Cette longueur étant beaucoup plus grande que l'épaisseur de la gaine, il n'y aura pas de collisions à l'intérieur de celle-ci. Avec $d=10^{-3} \mathrm{~cm}$ et une différence de potentiel de $45 \mathrm{~V}$ le champ électrique moyen est de $45 \mathrm{kV} / \mathrm{cm}$ à l'intérieur de la gaine. Avec de tels champs on pourrait trouver une augmentation de l'émission thermoélectronique par effet de champ. Une étude expérimentale a montré que cet effet pouvait être négligé car dans le cas le plus défavorable on ne trouve qu'une augmentation de $10 \%$ de l'émission [8].

5.3 IONISATION ET EXCITATION DANS LA CATHODE. Abstraction faite de la gaine, à l'intérieur de la cathode le champ électrique est faible de sorte que la probabilité, pour que des électrons provenant de l'ionisation d'atomes d'argon acquièrent une énergie suffisante pour produire une ionisation à leur tour est faible. Celle-ci est donc produite essentiellement par les électrons primaires émis par la paroi. Une étude théorique des processus de perte d'énergie des électrons dans la cathode a été faite par Ferreira [9]. Nous avons vu (Fig. 2) que dans la cathode la densité des neutres décroissait lorsqu'on allait vers l'aval. Les libres parcours de collisions élastiques et d'ionisation augmentent donc lorsqu'on se déplace dans le sens de l'écoulement. A l'arrière de la zone active les ions sont produits près de la paroi tandis que près du plan de sortie ils sont produits dans tout le volume de la cathode. Nous avons signalé qu'il existait une limite aval pour les mesures par sondes. Cette limite correspond à l'abscisse pour laquelle le libre parcours d'ionisation est égal au rayon de la cathode.

5.4 COURANTS D'IONS ET D'ÉLECTRONS A LA SURFACE DE LA CATHODE. - La température de la paroi de la cathode étant connue, le courant thermoélectronique $I_{-}$est calculé à partir de la loi de Richardson. Il faut tenir compte dans le calcul de ce que la température de la paroi varie avec l'abscisse. Dans le cas que nous avons pris comme exemple ce courant $I_{-}$est égal à 2 A. Il faut noter que les deux faces de la cathode émettent des électrons et que le courant total émis par celle-ci est $2 I_{-}$.

Le courant d'ions $I_{+}$qui arrive sur la cathode est égal au courant d'ions à la limite plasma-gaine où $j_{+}=n_{+} q v_{+}=0,84 \mathrm{~A} / \mathrm{cm}^{2}$ pour $T_{+}=2000^{\circ}$. Pour la colonne de plasma dont la longueur est de $45 \mathrm{~mm}$ et le diamètre de $5,6 \mathrm{~mm}, I_{+}=6,3 \mathrm{~A}$. Si pour les électrons il faut prendre en compte le courant sur les deux faces de la cathode, en ce qui concerne les ions, sur la face externe de la cathode la pression est 100 fois plus faible qu'à l'intérieur, le courant est donc négligeable. Le courant total I à la surface de la cathode doit donc être égal à $I=2 I_{-}+I_{+}=10,3$ A. Le modèle très simple que nous avons défini fournit donc une valeur très proche de celle qui a été mesurée et qui, dans ce cas, est de $10 \mathrm{~A}$. Il faut cependant remarquer qu'une augmentation de $50^{\circ}$ de la température de la cathode multiplierait par 1,6 le courant électronique, et qu'étant données les approximations que suppose ce modèle il serait illusoire de penser obtenir mieux qu'une estimation des courants.

5.5 MÉCANISMES D'ÉCHAUFFEMENT ET DE REFROIDISSEMENT DE LA PAROI DE LA CATHODE. - Une étude détaillée des processus d'échauffement et de refroidissement est présentée dans [7] où il est montré que, pour l'échauffement, les processus dominants sont le bombardement par les ions et le bombardement par les métastables. Pour le refroidissement, les processus dominants sont le rayonnement et la conduction thermique par le métal vers le support de la cathode.

- Echauffement par les ions : les ions apportent sur la paroi l'énergie cinétique qu'ils ont acquise dans la gaine et l'énergie de recombinaison avec les électrons du métal. La puissance ainsi apportée s'écrit :

$$
\begin{aligned}
& P_{+}=I_{+}\left(\Delta v_{\mathrm{g}}+n_{\mathrm{i}}-v_{\mathrm{s}}\right) \\
& P_{+}=6,3 \times 56,6=356 \mathrm{~W}
\end{aligned}
$$

$\Delta v_{\mathrm{g}}=$ chute de potentiel dans la gaine,

$v_{\mathbf{i}}=$ potentiel d'ionisation $=15,75 \mathrm{~V}$ pour l'argon, $v_{\mathrm{s}}=$ travail de sortie $=4,12 \mathrm{~V}$ pour le tantale.

- Echauffement par les métastables : la température des atomes excités étant égale à celle de la paroi il n'y aura pas d'échange de chaleur par conduction. Par contre les atomes métastables céderont leur énergie d'excitation $(11,6 \mathrm{eV})$ à la paroi. La densité des métastables est inférieure à $10^{13} \mathrm{~cm}^{-3}$ et l'énergie cédée est plus faible que pour les ions. On peut négliger leur apport d'énergie devant celui des ions.

- Pertes par rayonnement : l'énergie rayonnée $P_{\mathbf{r}}$ est égale à :

$$
\begin{gathered}
P_{\mathrm{r}}=\varepsilon(T) \sigma T^{4} \\
\sigma=\underset{\substack{\text { phan }\\
}}{5,67 \times 10^{-12} \mathrm{~W} \mathrm{~cm}^{-2} \mathrm{~K}-\text { constante de Ste- }} \\
\varepsilon(T)=0,05+1,05 \times 10^{-4}\left(T-T_{0}\right) \text { pour le tantale. }
\end{gathered}
$$


Dans l'exemple que nous considérons on trouve $P_{\mathrm{r}}=365 \mathrm{~W}$.

- Pertes par conduction : la cathode se refroidit par conduction vers son support. La puissance transmise par conduction à travers le métal est égale à :

$$
\begin{aligned}
& P_{\mathrm{c}}=\lambda s \frac{\mathrm{d} T}{\mathrm{~d} x} \\
& \lambda \quad=0,54 \mathrm{~W} / \mathrm{cm}^{2}\left({ }^{\circ} \mathrm{C}\right) \mathrm{cm}^{-1}, \\
& s \quad=\text { section de la cathode, }
\end{aligned}
$$

$\mathrm{d} t / \mathrm{d} x=$ gradient de température égal à $1000 \% / \mathrm{cm}$ à la limite amont de la zone active.

$$
P_{\mathrm{c}}=20 \mathrm{~W} \text {. }
$$

Les pertes représentent donc $385 \mathrm{~W}$ alors qu'on a trouvé $356 \mathrm{~W}$ pour le terme d'apport d'énergie. Le bon accord entre les 2 termes de ce bilan est une justification du modèle choisi. Il faut cependant noter que ce calcul utilise des grandeurs déduites de mesures (courant d'ions, d. d. p. dans la gaine). Les réserves faites, dans le cas du courant, sur la précision de cellesci restent valables ici.

6. Conclusion. - L'étude des paramètres de l'écoulement a permis de déterminer la répartition de pression de l'argon à l'intérieur de la cathode. Les mesures faites au moyen de sondes électrostatiques montrent que la colonne de plasma pénètre dans toute la zone active de la cathode. Cette colonne de plasma est à un potentiel inférieur de quelques volts à celui de l'anode et la densité y est élevée, de l'ordre de $10^{14}$ électrons/ $\mathrm{cm}^{3}$. Près de la paroi de la cathode existe une gaine dans laquelle la chute de potentiel est voisine de la tension de décharge. A l'intérieur de la cathode le champ électrique axial est faible et presque toute la chute de tension est concentrée à la limite amont de la zone active. L'ionisation de l'argon est produite essentiellement par les électrons thermoélectroniques émis par la cathode et accélérés dans cette gaine.

Les mesures faites à l'intérieur de la cathode ont permis de définir un modèle très simple pour la colonne de plasma dans la zone active. Les courants calculés sont en très bon accord avec les valeurs expérimentales. On trouve de même des valeurs très voisines lorsqu'on calcule avec ce modèle la perte d'énergie par la cathode et l'apport d'énergie à la surface de celle-ci. Les études expérimentales ont été faites avec une cathode de $6 \mathrm{~mm}$ de diamètre mais le modèle établi reste valable tant que le courant de décharge est suffisant pour qu'il y ait émission thermoélectronique de la cathode, que la condition sonique est satisfaite à l'extrémité de celle-ci et que le libre parcours d'ionisation est inférieur au diamètre.

\section{Bibliographie}

[1] LuCE, J. S., Proc. 2nd UN Conf. on Peaceful uses of Atomic Energy 31 (1958) 305, Geneva.

[2] Lidsky, L. M., Rothleder, S. D., Rose, D. J., Yoshikawas, S., Michelson, C. and Mackin, R. J., J. Appl. Phys. 33 (1962) 2490.

[3] Boeschoten, F., Kowen, R., Sens, A., XII C. I. P. I. G. (1975) 98, Eindhoven.
[4] Brunet, A., Conf. on Hollow Cathode Discharges (1971) Orsay.

[5] BRUnet, A., Thèse (1975), Orsay.

[6] Delcrorx, J. L., Physique des Plasmas II (Dunod, Paris) 1966.

[7] Trindade, A. R., Thèse (1970), Orsay.

[8] Willins, D. J. and Boyd, R. L. F., J. Phys. D Appl. Phys. 6 (1973) 1447

[9] Matos Ferreira, C., Thèse (1976), Orsay. 